\title{
ARTICLE
}

Minimal residual disease

\section{Clinical impact of panel-based error-corrected next generation sequencing versus flow cytometry to detect measurable residual disease (MRD) in acute myeloid leukemia (AML)}

\author{
Nikhil Patkar $\mathbb{D}^{1,2} \cdot$ Chinmayee Kakirde ${ }^{1} \cdot$ Anam Fatima Shaikh ${ }^{1} \cdot$ Rakhi Salve $^{1} \cdot$ Prasanna Bhanshe $^{1}$. \\ Gaurav Chatterjee $^{1,2} \cdot$ Sweta Rajpal $^{1,2} \cdot$ Swapnali Joshi $^{1} \cdot$ Shruti Chaudhary $^{1} \cdot$ Rohan Kodgule $^{1} \cdot$ Sitaram Ghoghale $^{1}$ • \\ Nilesh Deshpande ${ }^{1}$ - Dhanalaxmi Shetty ${ }^{3}$ Syed Hasan Khizer ${ }^{3,4}$ - Hasmukh Jain ${ }^{3,4}$ - Bhausaheb Bagal ${ }^{2,4}$. \\ Hari Menon ${ }^{5} \cdot$ Navin Khattry $\mathbb{B}^{3,5} \cdot$ Manju Sengar ${ }^{2,4} \cdot$ Prashant Tembhare $\mathbb{C}^{1,2} \cdot$ Papagudi Subramanian $\mathbb{D}^{1,2} \cdot$ \\ Sumeet Gujral ${ }^{1,2}$
}

Received: 26 August 2020 / Revised: 30 November 2020 / Accepted: 7 January 2021 / Published online: 8 February 2021

(c) The Author(s), under exclusive licence to Springer Nature Limited 2021. This article is published with open access

\begin{abstract}
We accrued 201 patients of adult AML treated with conventional therapy, in morphological remission, and evaluated MRD using sensitive error-corrected next generation sequencing (NGS-MRD) and multiparameter flow cytometry (FCM-MRD) at the end of induction (PI) and consolidation (PC). Nearly $71 \%$ of patients were PI NGS-MRD ${ }^{+}$and $40.9 \%$ PC NGS-MRD ${ }^{+}$ (median VAF $0.76 \%)$. NGS-MRD ${ }^{+}$patients had a significantly higher cumulative incidence of relapse $(p=0.003)$, inferior overall survival $(p=0.001)$ and relapse free survival $(p<0.001)$ as compared to NGS-MRD ${ }^{-}$patients. NGS-MRD was predictive of inferior outcome in intermediate cytogenetic risk and demonstrated potential in favorable cytogenetic risk AML. PI NGS-MRD ${ }^{-}$patients had a significantly improved survival as compared to patients who became NGS-MRD ${ }^{-}$ subsequently indicating that kinetics of NGS-MRD clearance was of paramount importance. NGS-MRD identified over 80\% of cases identified by flow cytometry at PI time point whereas FCM identified $49.3 \%$ identified by NGS. Only a fraction of cases were NGS-MRD ${ }^{-}$but FCM-MRD ${ }^{+}$. NGS-MRD provided additional information of the risk of relapse when compared to FCM-MRD. We demonstrate a widely applicable, scalable NGS-MRD approach that is clinically informative and synergistic to FCM-MRD in AML treated with conventional therapies. Maximum clinical utility may be leveraged by combining FCM and NGS-MRD modalities.
\end{abstract}

Supplementary information The online version contains supplementary material available at https://doi.org/10.1038/s41375021-01131-6.

Nikhil Patkar

nvpatkar@gmail.com

1 Haematopathology Laboratory, ACTREC, Tata Memorial Centre, Navi Mumbai, India

2 Homi Bhabha National Institute (HBNI), Mumbai, India

3 Dept of Cytogenetics, ACTREC, Tata Memorial Centre, Navi Mumbai, India

4 Adult Haematolymphoid Disease Management Group, Tata Memorial Centre, Mumbai, India

5 Haemato-Oncology, CyteCare Cancer Hospital, Bangalore, India

\section{Introduction}

Acute myeloid leukemia is a disease characterized by heterogeneous biology resulting in varying clinical outcomes including relapse $[1,2]$. There are limited novel treatment options such as targeted therapies using FLT3 or $I D H 2$ inhibitors and most patients are treated based on the presumptive risk of relapse [3, 4]. This risk- adapted therapy typically considers standard cytogenetic and molecular variables as recommended by the European Leukemia Net [5]. Based on this risk, patients are recommended treatment with conventional (induction and consolidation) regimens or offered intensive therapy such as allogeneic bone marrow transplantation (aBMT) after achievement of remission. Amongst these, the largest group remains as intermediate risk AML characterized by non-uniform clinical outcomes.

The monitoring of a patient's response to chemotherapy, called, measurable residual disease (MRD) is one of the 
most important predictors of outcome in hematological malignancies. Several investigators have demonstrated the clinical utility of flow cytometry based MRD detection (FCM-MRD) in AML at early chemotherapy time points as well as in a pre-transplant setting [6-14]. Although universally applicable, FCM-MRD suffers from suboptimal ability to predict relapse in AML compared to precursor B lineage acute lymphoblastic leukemia. A diverse array of sensitive molecular methods have been used to detect MRD in AML such as real time PCR [15] and droplet digital PCR [16]. These are useful for monitoring of individual gene mutations such as AML with mutated NPM1 [17, 18] and chimeric gene fusions such as RUNX1-RUNX1T1 [19]. Next generation sequencing (NGS) is a promising tool for sensitive MRD monitoring and has been used successfully to monitor NPM1 [20, 21], RUNX1, [22] and FLT3 [23] mutations as well as chimeric gene fusions [24, 25]. Amongst these, targets such as NPM1 or chimeric gene fusions are highly stable between diagnosis and relapse and not particularly vulnerable to clonal evolution.

DNA-based focused target enrichment strategies (gene panels) are an attractive solution to detect MRD using NGS (NGS-MRD) as they can be applied to a broader population of patients as compared to single gene molecular testing [26-29].

However, short read sequencers are inherently prone to base calling errors limiting variant calling at 3-5\% variant allele fraction (VAF) [30]. Although acceptable for diagnostic molecular pathology, this is undesirable assay performance for the detection of MRD. Error-corrected sequencing involves the physical incorporation of random oligonucleotides or unique molecular identifiers (UMI) at the library preparation stage prior to amplification of DNA. This allows us to tag individual DNA molecules with an unique molecular fingerprint [31, 32]. Such approaches have been used for myelodysplastic syndromes [33] and for pre-transplant MRD monitoring of AML as demonstrated by Thol and colleagues [34]. Thol utilized a sensitive patient-specific mutation tracking approach using UMIbased MRD detection. Although applicable to a broad spectrum of AML mutations, a tailored approach poses logistical and regulatory hurdles towards prospective MRD testing especially for early MRD time points.

In this study, we have evaluated the clinical utility of error-corrected NGS to detect MRD in AML using single molecule molecular inversion probes (smMIPS) [31, 35]. Each smMIP contains an $8 \mathrm{bp}$ UMI and binds to a single molecule of DNA. Using consensus sequence-based variant calling we can detect somatic mutations including small indels in a sensitive manner. We demonstrate that errorcorrected NGS-MRD at early time points in therapy is significantly predictive of outcome in patients of AML treated with conventional therapies. Furthermore, we systematically compare multicolor FCM-MRD with errorcorrected NGS-MRD and assess the clinical utility of these two assays in a cohort of AML.

\section{Methods}

\section{Patient characteristics, treatment and MRD sampling}

The study was approved by the institutional ethics committee (IEC-III project 163) and participants were accrued after informed consent. A total of 393 adult patients of AML, diagnosed as per 2008 WHO criteria, were accrued in this study over a period of 6 years (Feb 2013 to May 2019). Cytogenetic (FISH and karyotyping) workup was performed as previously described [9, 21]. Somatic mutations at diagnosis were evaluated using a smMIPS based 50 gene myeloid panel as described previously [36]. We describe an NGS-MRD approach that was applicable to more than $80 \%$ of patients in this AML cohort [327 out of 393 AMLs, median two mutations per case (range 1-6 trackable mutations); Supplementary Fig. 1]. Of those $(n=319)$ achieving morphological CR, the smMIPS MRD panel was applicable to $266(83.4 \%)$. MRD assessment could be performed in 201 adult patients of AML (enrollment flow chart in Supplementary Fig. 2). A summary of the clinical and laboratory characteristics of these 201 patients can be seen in Table 1.

All patients were treated with conventional " $3+7$ " induction chemotherapy and further treated with high dose cytarabine (HiDAC) or allogeneic bone marrow transplantation (aBMT), if feasible [36]. Only 15 patients received aBMT and their outcome was not different from the rest with respect to OS and RFS ( $p=$ not significant; Supplementary Fig. 3) and are not considered separately. Sample for FCM-MRD was obtained from the bone marrow at the end of induction (PI; $n=200$ ) and end of first consolidation cycle (PC, $n=98$ ). NGS-MRD sample also obtained at the same time points (PI-196; PC-127) from the bone marrow ( $n=269$; PI:181 and PC:88) or peripheral blood $(n=51$; PI:15 and PC:36).

\section{Detection of MRD using error-corrected NGS (NGS- MRD)}

We created a 34-gene panel comprising of a pool of 302 smMIPS (as seen in Supplementary Table 1). In brief, this panel covers regions of 34 commonly mutated genes in AML (ATM, BCOR, DNMT3A, EZH2, FLT3, GATA1, GATA2, IDH1, IDH2, JAK2, KDM6A, KIT, KMT2D, KRAS, NF1, NOTCH1, NOTCH2, NPM1, NRAS, PHF6, PTPN11, RAD21, RUNX1, SETBP1, SF3B1, SH2B3, SMC1A, 
Table 1 Summary of clinical, laboratory, and MRD characteristics of patients accrued in this study.

\begin{tabular}{|c|c|}
\hline Parameter & Observation $(\%)$ \\
\hline \multicolumn{2}{|l|}{ Demographics: } \\
\hline Age & $\begin{array}{l}\text { Range: } 18-63 \text { years Median: } \\
36 \text { years }\end{array}$ \\
\hline Sex & Male: Female: 1.48: 1 \\
\hline \multicolumn{2}{|l|}{ Clinical characteristics: } \\
\hline Total number of patients accrued & 201 \\
\hline \multicolumn{2}{|l|}{ Remission characteristics: } \\
\hline Complete remission (CR) & 31 \\
\hline $\begin{array}{l}\mathrm{CR} \text { with incomplete hematologic } \\
\text { recovery }(\mathrm{CRi})\end{array}$ & 170 \\
\hline \multicolumn{2}{|l|}{ Bone marrow transplantation: } \\
\hline Patients who underwent BMT & 15 \\
\hline \multicolumn{2}{|l|}{ Laboratory characteristics: } \\
\hline \multicolumn{2}{|l|}{ Blood counts at presentation } \\
\hline 1. More than $50,000 / \mathrm{mm}^{3}$ & 49 \\
\hline 2. Less than $50,000 / \mathrm{mm}^{3}$ & 152 \\
\hline \multicolumn{2}{|c|}{ Classification according to cytogenetic risk: } \\
\hline Favorable risk & $48(23.9 \%)$ \\
\hline Intermediate risk & $136(67.7 \%)$ \\
\hline Poor risk & $17(8.5 \%)$ \\
\hline \multicolumn{2}{|l|}{ Post Induction flow MRD $(n=200)$ : } \\
\hline MRD positive & $88(44.0 \%)$ \\
\hline MRD negative & $112(56.0 \%)$ \\
\hline \multicolumn{2}{|c|}{ Post consolidation flow MRD $(n=98)$ : } \\
\hline MRD positive & $21(21.4 \%)$ \\
\hline MRD negative & $77(78.6 \%)$ \\
\hline \multicolumn{2}{|c|}{ Post induction NGS-MRD $(n=196)$ : } \\
\hline MRD positive & $139(70.9 \%)$ \\
\hline MRD negative & $57(29.1 \%)$ \\
\hline \multicolumn{2}{|c|}{ Post consolidation NGS-MRD $(n=127)$ : } \\
\hline MRD positive & $52(40.9 \%)$ \\
\hline MRD negative & $75(59.1 \%)$ \\
\hline \multicolumn{2}{|c|}{$\begin{array}{l}\text { Comparative analysis of FCM-MRD and NGS-MRD (post induction } \\
n=195 \text { ) }\end{array}$} \\
\hline NGS-MRD $^{+}$FCM-MRD $^{+}$ & $68(34.9 \%)$ \\
\hline NGS-MRD $^{+}$FCM-MRD $^{-}$ & $70(35.9 \%)$ \\
\hline NGS-MRD $^{-}$FCM-MRD $^{+}$ & $17(8.7 \%)$ \\
\hline NGS-MRD $^{-}$FCM-MRD $^{-}$ & $40(20.5 \%)$ \\
\hline \multicolumn{2}{|c|}{$\begin{array}{l}\text { Comparative analysis of FCM-MRD and NGS-MRD (post } \\
\text { consolidation; } n=87 \text { ) }\end{array}$} \\
\hline NGS-MRD $^{+}$FCM-MRD $^{+}$ & $09(10.3 \%)$ \\
\hline NGS-MRD $^{+}$FCM-MRD ${ }^{-}$ & $28(32.2 \%)$ \\
\hline NGS-MRD $^{-}$FCM-MRD $^{+}$ & $08(9.2 \%)$ \\
\hline NGS-MRD $^{-}$FCM-MRD $^{-}$ & $42(48.3 \%)$ \\
\hline
\end{tabular}

SRSF2, STAG2, TET2, TP53, U2AF1, WT1, ZRSR2). The panel was rebalanced (Supplementary Fig. 4) to ensure uniform capture across regions. Approximately $600 \mathrm{ng}$ of genomic DNA was captured, treated with exonucleases, and PCR amplified to create a sequencing ready library. Details pertaining to smMIPS design, assay standardization, and sequencing are detailed in supplementary methods.

Reads were demultiplexed, trimmed, paired end assembled, and mapped to the human genome (build hg19). Singleton reads (originating from one UMI) were discarded, and consensus family-based variant calling performed using tools described in supplementary methods. We then created a site and mutation-specific error model to ascertain the relevance of an observed variant at each site [35]. Criteria for variant calling using the smMIPS MRD assay are described in supplementary methods. FLT3-ITD were detected using a novel one-step PCR-based NGS assay (see Supplementary Table 3). Variants were detected using a recently described algorithm for the accurate detection of FLT3-ITD [37]. NPM1 mutations were additionally tracked using an ultrasensitive orthogonal NPM1 MRD assay [21].

\section{Detection of MRD using multicolour flow cytometry (FCM-MRD)}

FCM-MRD was detected using a previously described 10color two-tube MRD assay [9, 21, 36, 38]. This approach uses a combination of leukemia associated immunophenotype and difference from normal approaches to detect MRD in AML.

\section{Endpoints and statistical analyses}

Overall survival (OS) and relapse free survival (RFS) were calculated as previously described $[9,21,36]$. The prognostic impact of NGS and FCM-MRD assays on OS and RFS was computed using the Kaplan-Meier method and compared using log-rank test. Prognostic relevance of individual gene mutations seen at baseline in patients of AML were assessed and variables found to be significantly predictive of outcome were included in multivariate analysis. Multivariate analysis was performed using the cox proportional-hazards regression analysis that considered FCM-MRD and NGS-MRD. Separate models were constructed for post induction and post consolidation MRD time points. Grey test was used to compare the cumulative incidences of relapse (CIR) and non-relapse mortality (NRM) using "cmprsk" module in R [39]. The same module was used to generate representative graphs. Competing risk regression modeling was performed using cause-specific hazard approach to determine the different rates of relapse in the presence of covariates [40]. Positive predictive (PPV) and negative predictive value (NPV) were calculated as described in [34]. Accuracy was calculated using the formula: $\quad$ accuracy $=$ sensitivity $\times$ prevalence + specificity $\times$ (1-prevalence) [41]. 


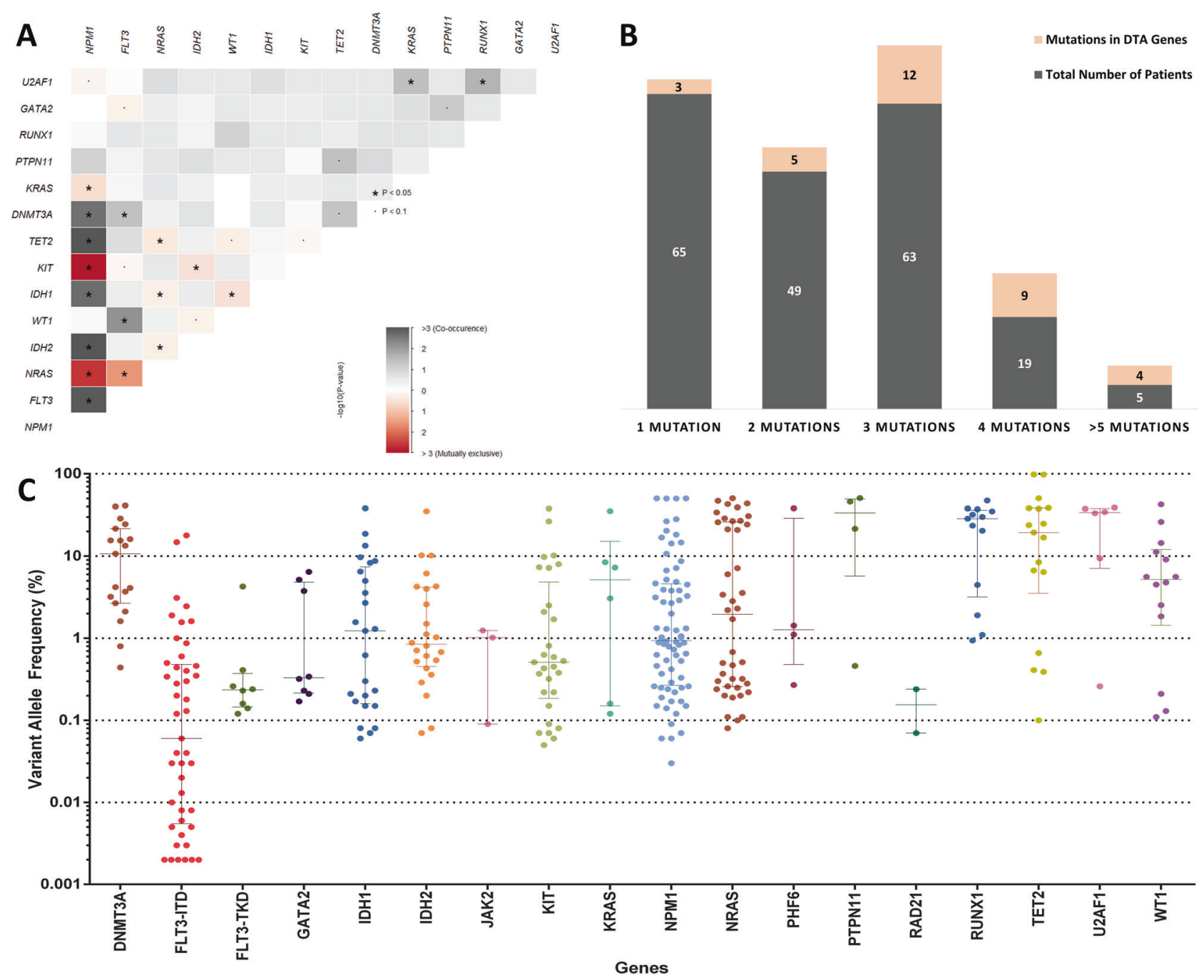

Fig. 1 Somatic mutations in AML detected at diagnosis and during therapy. A The interaction of mutations at baseline is demonstrated here using Fisher's exact test. Co-occurrence is indicated in gray color and mutual exclusivity is indicated in red. B The total number of mutations detected per patient and the number of such patients in the

\section{Results}

The median follow-up of the cohort was 42.3 months. The median OS was 35.9 months (95\% CI 27.2-42.8) and median RFS was 21.6 months (95\% CI 17.0-28.9) months. Additional patient characteristics can be seen in Table 1.

\section{Next generation sequencing based AML MRD detection}

We describe an NGS-MRD approach that was applicable to more than $80 \%$ of patients in this AML cohort $[83.4 \%(n=$ 266) patients in morphological CR]. A co-occurrence plot indicating interactions of mutations tracked by NGS-MRD prior to therapy, can be seen in Fig. 1A. The applicability of cohort is displayed. The total number of mutations in DNMT3A-TET2ASXL1 genes is indicated here as a fraction. C Variant allele frequencies of mutations detected at MRD time points for patients of AML in morphological remission. The bars indicate median values with interquartile ranges.

this MRD panel, when patients $(n=201)$ are classified by cytogenetic risk is seen in supplementary Table 2 . In a limit of detection experiment (Supplementary Fig. 5), we demonstrated that we could detect leukemic clones till a lower limit of $0.05 \%$ ( $0.03 \%$ for NPM1 mutation). Error modeling of normal patients indicated a higher prevalence of $\mathrm{C}>\mathrm{T}$ and $\mathrm{G}>\mathrm{A}$ changes consistent with oxidative DNA damage (Supplementary Fig. 6) [35]. FLT3-ITD could be detected at a limit of $0.002 \%$ VAF (Supplementary Fig. 7). For smMIPS-based MRD, sequencing was performed at a median coverage of $14,728 \mathrm{x}$ (11,363x consensus coverage) whereas, for FLT3-MRD assay, the median coverage was 1,396,366x. A total of 344 mutations could be detected in 323 MRD samples (Fig. 1B, C) with a median VAF of $0.95 \%$ [0.76\% after exclusion of mutations in DNMT3A, TET2, ASXL1 (DTA) genes]. A median of two mutations 
could be detected per patient (range 1-4) at the end of induction.

Nearly $71 \%(n=139 ; 70.9 \%)$ of patients were NGS$\mathrm{MRD}^{+}$at the end of induction and $40.9 \%(n=52)$ at the end of consolidation. NGS-MRD ${ }^{+}$patients had a significantly higher CIR, OS, and RFS as compared to NGS$\mathrm{MRD}^{-}$patients at both MRD time points as seen in Fig. 2 and Tables 2, 3. The clinical impact of NGS-MRD when sample type was restricted to either the bone marrow (BM) or peripheral blood (PB) can be seen in Supplementary Figs. 8 and 9. The presence NGS-MRD was highly predictive of inferior OS and RFS for both MRD time points when patient samples were sourced from the BM. A similar trend can be postulated in PB sourced samples at PC time point but a definitive conclusion cannot be drawn due to limited numbers. NGS-MRD ${ }^{+}$patients demonstrated an inferior outcome in intermediate cytogenetic risk (and a tendency in favorable risk) as seen in Supplementary Fig. 10.

Out of 122 patients in whom both (PI and PC) MRD time points were assessed, 83 patients were PI NGS-MRD ${ }^{+}$and 46 were PC NGS-MRD ${ }^{+}$. A change in MRD profile occurred in 18 patients $(39.13 \%)$. This included a loss of mutation in most cases $(n=14)$ and gain in the rest (Supplementary Fig. 11). There were five patients who were NGS-MRD $^{-}$at the end of induction but became NGS-MRD ${ }^{+}$at end of consolidation. Of these, relapse was seen in two patients. It should be noted that this change in MRD mutation profile between two MRD time points is not conceptually the same as a genuine gain in mutation which was not present at diagnosis of AML. Patients who were NGS-MRD $^{-}$at all MRD time points had a significantly improved OS [HR 0.45; 95\% CI 0.22-0.9; $(p=0.02)$ ] and RFS [HR 0.49; 95\% CI 0.27-0.89; $(p=0.01)$ ] as compared to patients who became negative at the end of consolidation (Supplementary Fig. 12). Similarly, patients who were persistently NGS-MRD ${ }^{+}$had a significantly inferior outcome as compared to patients who were MRD negative at both time points (Supplementary Fig. 13). There was no genetic difference observed between these two groups (Supplementary Fig. 14).

We could detect MRD in NPM1 mutated AML using an orthogonal technique in 75 patients (23.2\% of all samples; Supplementary Fig. 15). There was a good correlation observed with NPM1 NGS-MRD assay $\left(R^{2}=0.71\right)$ at the limit of detection of the smMIPS MRD assay.

\section{FCM based AML MRD and comparison with NGS- MRD}

The presence of FCM-MRD was associated with inferior OS, RFS, and CIR at the end of induction and consolidation as detailed in Tables 2, 3 and Supplementary Fig. 16. On incorporating results combining both the MRD modalities, patients that were positive by both techniques $\left(\mathrm{FCM}^{+} \mathrm{NGS}^{+}\right)$had a significantly inferior outcome with respect to OS, RFS, and CIR at any MRD time point as compared to patients negative by both modalities as seen in Table 2 and supplementary Table 5 (Fig. 3). A comparison of the baseline mutational profiles between dual PI MRD positive $\left(\mathrm{FCM}^{+} \mathrm{NGS}^{+}\right)$and negative groups $\left(\mathrm{FCM}^{-} \mathrm{NGS}^{-}\right)$ revealed a significantly higher $(p=0.04)$ prevalence of RUNX1 mutations in the dual MRD positive subset (Supplementary Fig. 17). A total of 20 patients were $\left(\mathrm{FCM}^{+}\right.$ $\mathrm{NGS}^{-}$) assessed at PI and/or PC time points. Their genetic profiles as well as MRD results (FCM/NGS) and eventual outcome are detailed in supplementary Table 6 .

A total of 32 samples were sourced from PB for NGSMRD (PI:15, PC:17) where FCM-MRD was measured in BM. Of these, 14 were FCM-MRD but were NGS-MRD ${ }^{+}$. Meanwhile, three patients were FCM-MRD ${ }^{+}$but NGS$\mathrm{MRD}^{-}$as detailed in supplementary Table 7.

\section{Metrics for assay performance}

The PPV and NPV metrics of end of induction NGS-MRD to predict relapse in AML were $70.5 \%$ and $57.89 \%$ respectively with an accuracy of $66.84 \%$. FCM-MRD metrics at the end of induction were comparable for PPV (75\%), NPV (48.2\%), and accuracy to predict relapse (60\%). At the PI time point, NGS-MRD identified $80 \%$ (68 out of 85) of cases classified as MRD positive by FCM, whereas FCM identified just 68 out of 138 cases (49.3\%) identified by NGS. A detailed comparison of PPV, NPV, and accuracy of combinations of patients detected between these two assays can be seen in supplementary Table 8 .

\section{Multivariate analysis}

Multivariate analysis included high VAF (>11) FLT3-ITD, RUNXI mutation, poor risk cytogenetics along with FCM and NGS-MRD. The presence of high VAF FLT3-ITD, RUNX1 mutation, FCM-MRD, and NGS-MRD were important in predicting outcome as seen in Table 4 for OS and RFS at the post induction MRD time point.

\section{Discussion}

Recently, Hourigan and colleagues [42] performed ultradeep sequencing using a 13-gene panel to detect MRD in AML. In a pioneering effort, they demonstrated an advantage of myeloablative conditioning in preventing relapse in an AML cohort based on NGS-MRD results. The authors, however, were unable to compare their results with other 


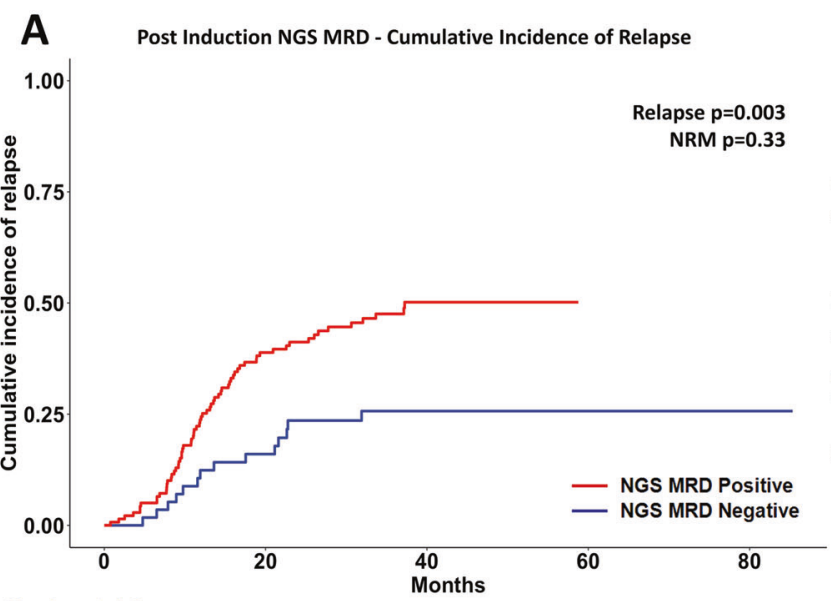

B Post Consolidation NGS MRD - Cumulative Incidence of Relapse
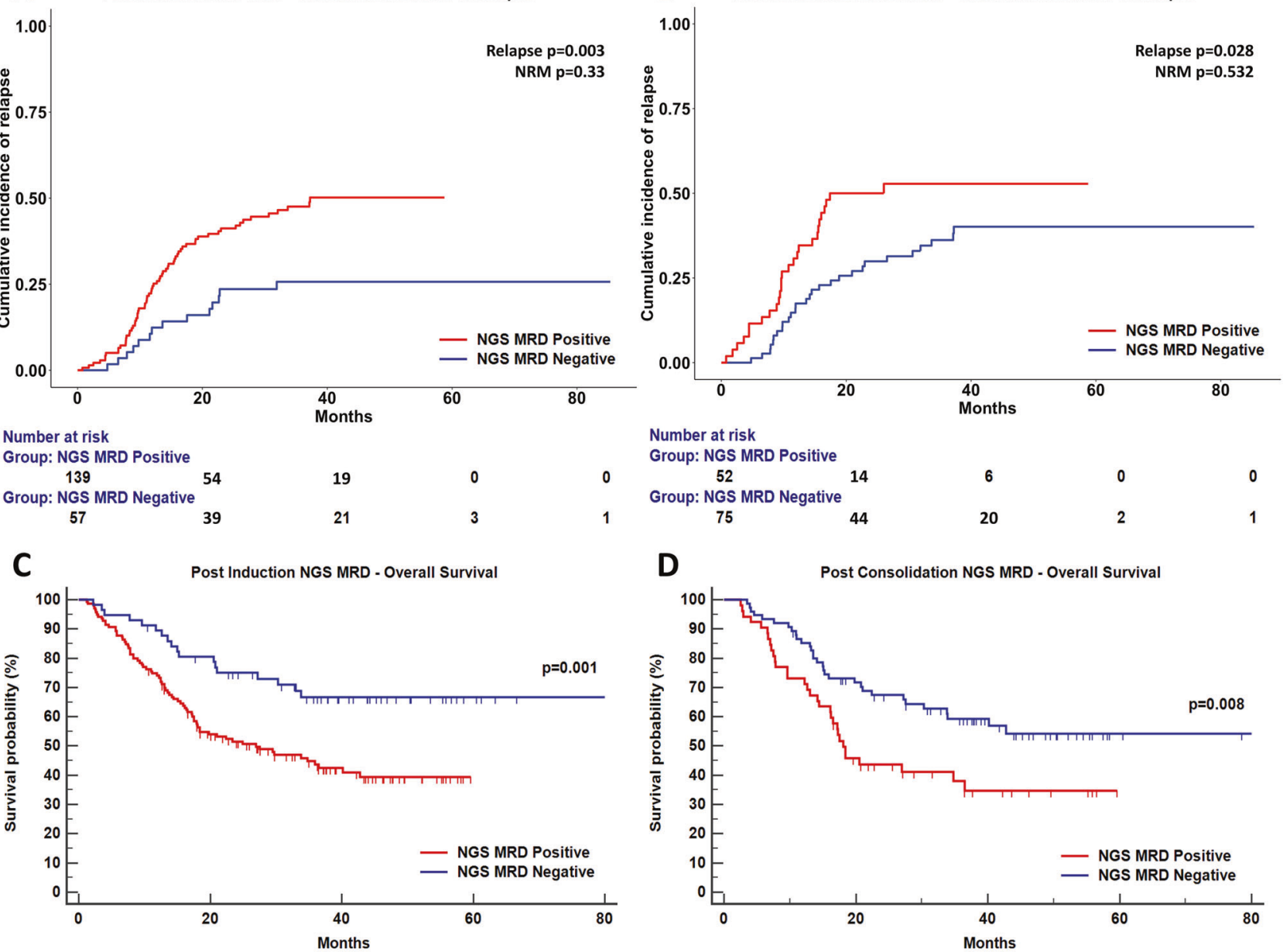

Number at risk

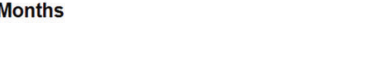

D
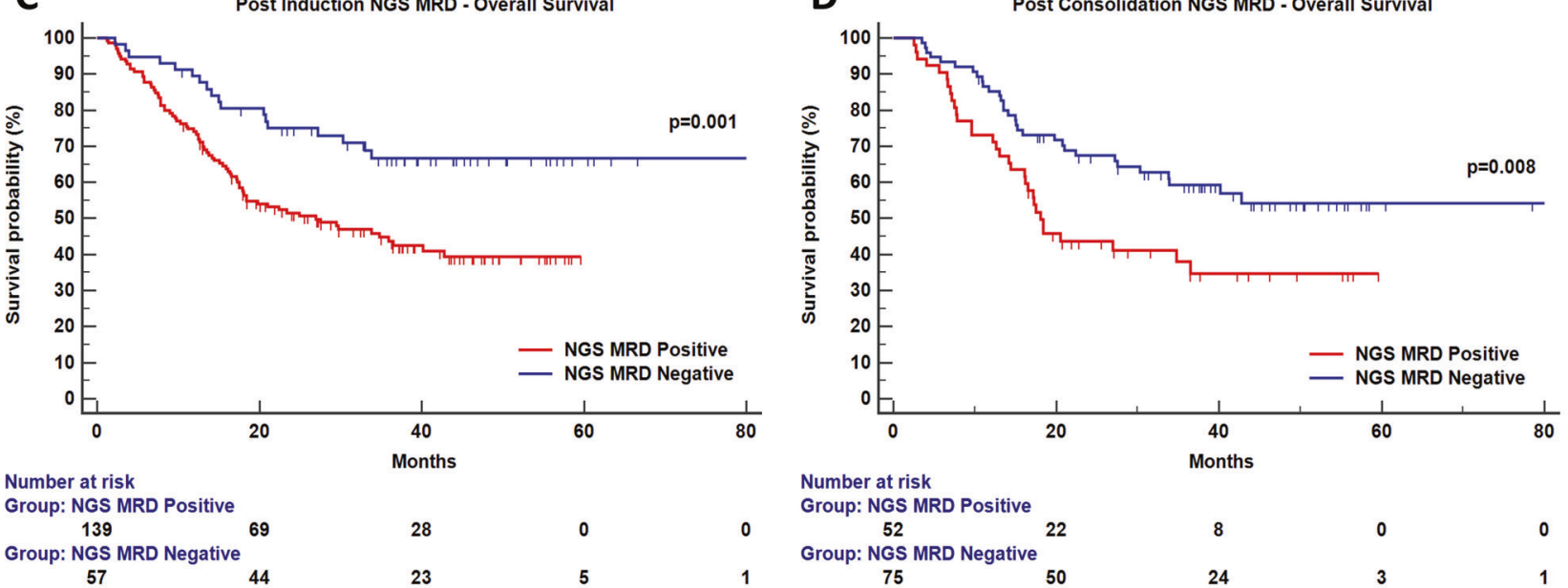

Number at risk

Group: NGS MRD Positive

52
Group: NGS MRD Negative

75
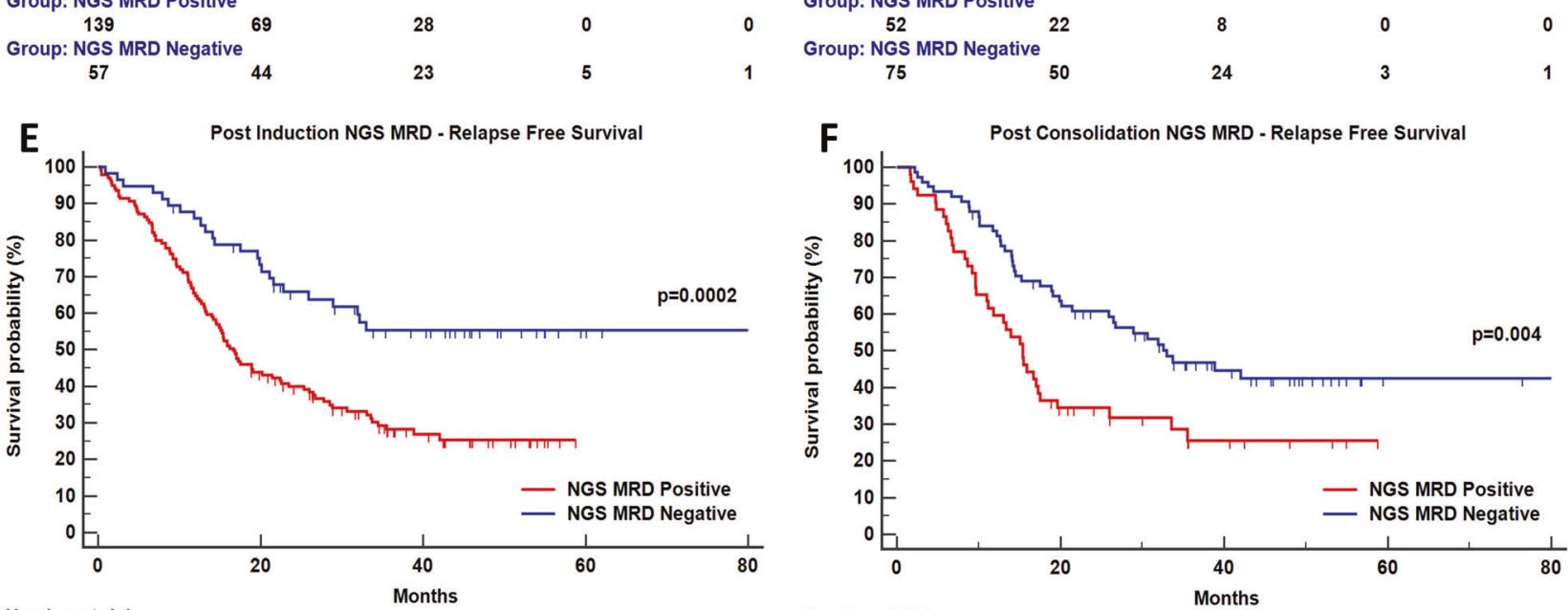

Number at risk

Group: NGS MRD Positive

$\begin{array}{ccccc}139 & 59 & 20 & 0 & 0 \\ \begin{array}{c}\text { Group: NGS MRD Negative } \\ 57\end{array} & 40 & 21 & 3 & 1\end{array}$

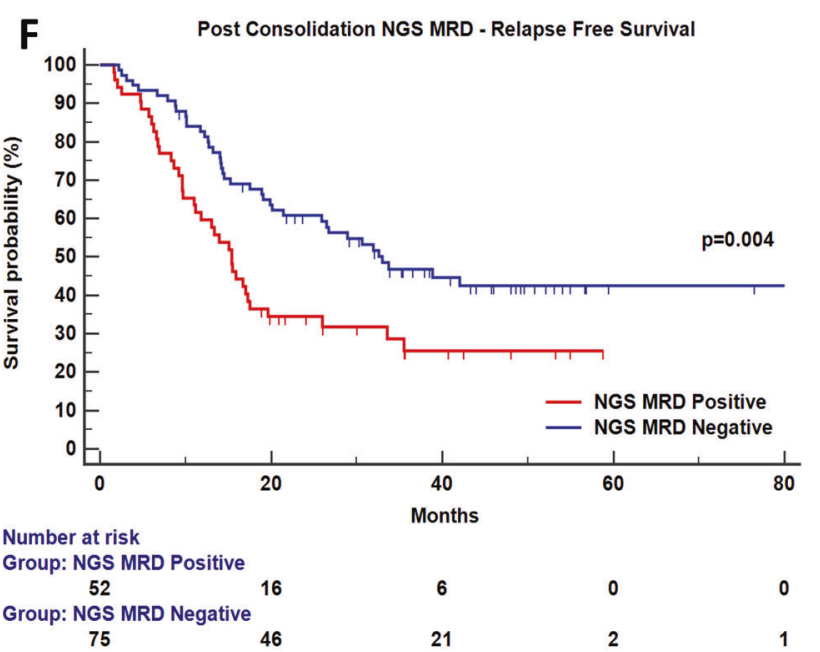

Fig. 2 Clinical relevance of error-corrected NGS-MRD. Presence of NGS-MRD at post induction (A) and post consolidation time points (B) is associated with a higher cumulative incidence of relapse (CIR).

Kaplan-Meyer plots indicate the clinical relevance of NGS-MRD with respect to OS and RFS at post induction $(\mathbf{C}, \mathbf{E})$ and post consolidation time points $(\mathbf{D}, \mathbf{F})$. 
Table 2 Prognostic influence of NGS-MRD, FCM-MRD and a combination of these modalities on the cumulative incidence of relapse (CIR).

Cumulative incidence of relapse

Post induction NGS-MRD

\begin{tabular}{l}
$\begin{array}{l}\text { Cumulative incidence of } \\
\text { relapse (CIR) }\end{array}$ \\
\hline HR 3-year CIR $(95 \% \mathrm{CI}) \quad P$ value
\end{tabular}

Non-relapse mortality (NRM)

HR 3-year cumulative incidence

$P$ value $(95 \% \mathrm{CI})$

MRD negative
MRD positive

Post consolidation NGS-MRD

$1 \quad 25.7 \%(14.9-37.9)$

$0.0005 \quad 1$

$18.7 \%(9.5-30.3)$

0.1676

$2.67 \quad 47.5 \%(38.7-55.8)$

$1.6323 .4 \%(16.6-30.8)$

Cumulative incidence of relapse Non-relapse mortality

HR 3-year CIR (95\% CI) $P$ value $\quad$ HR 3 -year cumulative incidence $\quad P$ value

MRD negative

(95\% CI)

MRD positive

Post induction FCM-MRD

$1 \quad 36.2 \%(25.1-47.4) \quad 0.007$

$16.6 \%(9.0-26.1)$

$2.0352 .8 \%(37.8-65.7) \quad 1.57 \quad 19.2 \%(9.8-31.0)$

Cumulative incidence of relapse Non-relapse mortality

HR 3-year CIR $(95 \% \mathrm{CI}) \quad P$ value $\quad$ HR 3 -year cumulative incidence $\quad P$ value (95\% CI)

MRD negative
MRD positive
Post consolidation FCM-MRD

MRD negative

MRD positive

Comparative analysis of FCM-MRD and NGS-MRD (post induction)

\section{NGS-MRD $^{-}$FCM-MRD $^{-}$ \\ NGS-MRD $^{+}$FCM-MRD ${ }^{-}$ \\ NGS-MRD $^{-}$FCM-MRD $^{+}$ \\ NGS-MRD $^{+}$FCM-MRD $^{+}$}

Comparative analysis of FCM-MRD and NGS-MRD (post consolidation)

\section{NGS-MRD $^{-}$FCM-MRD ${ }^{-}$ \\ NGS-MRD $^{+}$FCM-MRD ${ }^{-}$ \\ NGS-MRD $^{-}$FCM-MRD $^{+}$ \\ NGS-MRD ${ }^{+}$FCM-MRD ${ }^{+}$}

Dual time point NGS-MRD

MRD negative

Either MRD positive

MRD positive
$1 \quad 33.6 \%(24.7-42.7)$

$0.0004 \quad 1 \quad 19.8 \%(12.7-28.0)$

$2.17 \quad 49.8 \%(38.6-59.9)$

$1.63 \quad 25.2 \%(16.6-34.8)$

Cumulative incidence of relapse Non-relapse mortality

HR 3-year CIR $(95 \%$ CI) $P$ value $\quad$ HR 3-year cumulative incidence $P$ value $(95 \% \mathrm{CI})$

$1 \quad 35.9 \%(25.1-46.9) \quad 0.0005 \quad 1 \quad 19.9 \%(11.7-29.7)$

$3.0961 .9 \%(36.3-79.7) \quad 1.86 \quad 23.8 \%(8.2-43.9)$

0.1618

Cumulative incidence of relapse Non-relapse mortality

HR 3-year CIR $(95 \% \mathrm{CI}) \quad P$ value $\quad$ HR 3 -year cumulative incidence $P$ value $(95 \% \mathrm{CI})$

$1 \quad 18.7 \%(8.0-32.7) \quad 0.0001 \quad 1 \quad 13.8 \%(4.8-27.3)$

$3.36 \quad 41.9 \%(29.6-53.7) \quad 2.34 \quad 23.7 \%(14.2-34.6)$

$4.04 \quad 41.2 \%(17.5-63.6) \quad 3.07 \quad 29.4 \%(9.8-52.4)$

$5.40 \quad 52.5 \%(39.6-63.9) \quad 2.65 \quad 23.5 \%(14.2-34.2)$

Cumulative incidence of relapse Non-relapse mortality

HR 3-year CIR $(95 \%$ CI) $P$ value $\quad$ HR 3 -year cumulative incidence $\quad P$ value $(95 \% \mathrm{CI})$

$1 \quad 24.8 \%(12.6-39.0) \quad 0.0001 \quad 1 \quad 22.0 \%(10.7-35.9)$

0.1477

$2.55 \quad 50.0 \%(29.9-67.1) \quad 1.07 \quad 17.8 \%(6.3-34.1)$

$5.3987 .5 \%(17.2-98.9) \quad-\quad 0.0 \%(-)$

$5.80 \quad 55.5 \%(14.7-83.5)$

$3.2633 .3 \%(5.7-65.6)$

Cumulative incidence of relapse Non-relapse mortality

HR 3-year CIR (95\% CI) $P$ value HR 3 -year cumulative incidence $\quad P$ value (95\% CI)

$1 \quad 27.8 \%(13.5-44.0) \quad 0.01 \quad 1 \quad 15.9 \%(5.6-31.1)$

$2.1444 .7 \%(28.6-59.7) \quad 1.35 \quad 19.0 \%(8.8-32.2)$

$2.90 \quad 52.9 \%(37.0-66.6) \quad 1.63 \quad 17.4 \%(8.0-29.7)$
MRD assessment techniques. Here, we have assessed MRD at serial time points and have compared our results with 10color FCM-MRD, which is a widely used technique for the assessment of response to chemotherapy [43]. We find that NGS-MRD is comparable in applicability and adds value, especially when a clear distinction of regenerating myeloid progenitors from leukemic blasts is absent.
In our manuscript we demonstrate that NGS- MRD identified over $80 \%$ of cases identified by flow cytometry at PI time point. On evaluating 17 discrepant cases (PI time point; 15 BM samples) which were NGS-MRD ${ }^{-}$but FCM$\mathrm{MRD}^{+}$, we observed that majority patients (10 out of 17) are alive or have died due to causes other than relapse indicating that these could have been false positives. 
Table 3 Difference in overall survival and relapse free survival between FCM-MRD, NGS-MRD.

Univariate cox analysis

Post induction FCM-MRD
MRD negative
MRD positive
Post induction FCM-MRD
MRD negative
MRD positive
Post consolidation FCM-MRD
MRD negative
MRD positive

Post consolidation FCM-MRD

MRD negative

MRD positive

Post induction NGS-MRD

MRD negative

Overall survival (OS)

HR $(95 \%$ CI $)$

1

$2.1(1.40-3.13)$

Overall survival (OS)

HR $(95 \%$ CI)

Mean OS: 58.0 months; 95\% CI (51.2-64.8 months), Median OS: Not reached

Mean OS: 36.3 months; 95\% CI (29.6-43.0 months), Median OS: 18.4 months; 95\% CI (15.1-33.9 months)

Overall survival (OS)

\section{HR (95\% CI)}

1

$1.9(0.90-3.91)$

Overall survival (OS)

HR (95\% CI)

Mean OS: 52.6 months; 95\% CI (44.6-60.7 months), Median OS: Not reached

Mean OS: 28.7 months; 95\% CI (18.7-38.7 months), Median OS: 16.5 months; 95\% CI (11.2-33.9 months)

Overall survival (OS)

HR (95\% CI)

1

MRD positive

Post induction NGS-MRD

MRD negative

MRD positive

Post consolidation NGS-MRD

MRD negative

MRD positive

Post consolidation NGS-MRD

MRD negative
$2.2(1.47-3.42)$

Overall survival (OS)

HR (95\% CI)

Mean OS: 63.2 months; Median OS: Not reached

Mean OS: 32.9 months; 95\% CI (28.9-36.9 months), Median OS: 27.0 months; 95\% CI (17.9-42.8 months)

Overall survival (OS)

HR $(95 \%$ CI) 1

1.9 (1.14-3.22)

Overall survival (OS)

HR (95\% CI)

Mean OS: 55.5 months; 95\% CI (47.2-63.7 months), Median OS: Not reached 95\% CI (54.4-72.0 months),

Relapse free survival (RFS)

HR $(95 \% \mathrm{CI}) \quad P$

1

0.0008

$1.8(1.26-2.60)$

Relapse free survival (RFS)

\section{$P$}

0.0002

HR (95\% CI)

$P$

0.0008 95\% CI (40.2-53.8 months), Median RFS: 32.2 months 95\% CI (22.4-42.0 months)

Mean RFS: 28.4 months; 95\% CI (22.6-34.3 months), Median RFS: 15.4 months; 95\% CI (12.7-20.1 months)

Relapse free survival (RFS) HR $(95 \%$ CI)

$P$ 0.04 1 $2.4(1.17-4.81)$

Relapse free survival (RFS)

$P$ 0.04

HR (95\% CI)

Mean RFS: 45.4 months; 95\% CI (37.5-53.3 months), Median RFS: 31.9 months 95\% CI (19.1-38.9 months)

Mean RFS: 19.4 months; 95\% CI (11.6-27.2 months), Median RFS: 11.8 months; 95\% CI (9.6-20.1 months)

Relapse free survival (RFS)

$P$

0.001

\section{$P$}

0.001 HR (95\% CI)

1

$2.3(1.58-3.31)$

Relapse free survival (RFS) HR $(95 \%$ CI)

Mean OS: 54.9 months; 95\% CI (45.7-64.2 months), Median OS: Not reached

Mean OS: 26.1 months; 95\% CI (22.4-29.6 months), Median OS: 16.7 months; 95\% CI (14.5-22.4 months) Relapse free survival (RFS)

$P$

0.008

\section{$P$}

0.008
HR $(95 \%$ CI $)$

1

1.9 (1.18-3.06)

Relapse free survival (RFS) HR (95\% CI)

Mean OS: 46.9 months; 95\% CI (38.8-54.9 months), Median OS: 33.0 months; 95\% CI (21.4-42.0 months)
0.001

$P$

$P$

0.0002

$P$

0.0002

$P$

0.004

$P$

0.004 
Table 3 (continued)

\begin{tabular}{llll}
\hline Univariate cox analysis & & \\
\hline Post induction FCM-MRD & Overall survival (OS) & & $\frac{\text { Relapse free survival (RFS) }}{$\cline { 2 - 2 }} \\
\cline { 2 - 3 } HR (95\% CI) & & HR (95\% CI) \\
\hline MRD positive & Mean OS: 30.1 months; & Mean OS: 24.6 months; \\
& $95 \%$ CI (23.7-36.5 months), & 95\% CI (18.7-30.6 months), \\
& Median OS: 18.1 months; & Median OS: 15.4 months; \\
& $95 \%$ CI (16.1-36.5 months) & $95 \%$ CI (11.2-17.5 months) \\
\hline
\end{tabular}

$O S$ overall survival, $R F S$ relapse free survival, $C I$ confidence interval.
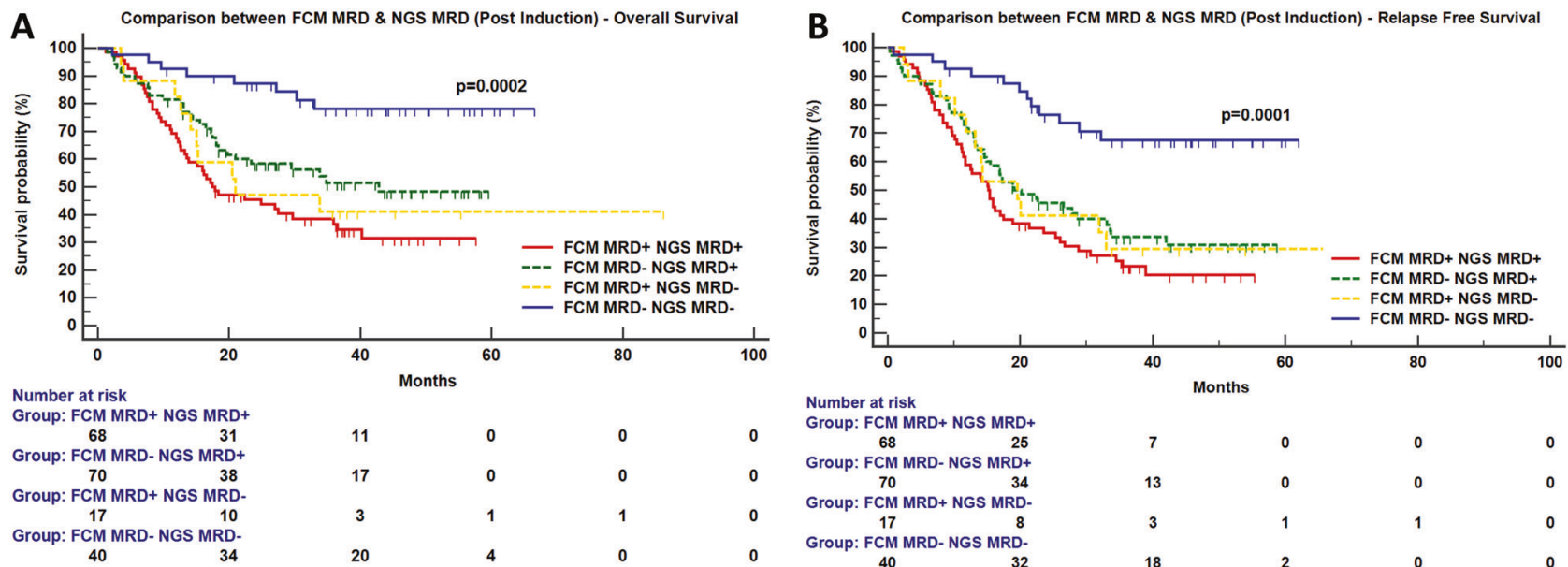

\section{Number at risk}

Group: FCM MRD+ NGS MRD+

$68 \quad 25$

Group: FCM MRD- NGS MRD+

Group: FCM MRD+ NGS MRD-

$\begin{array}{cr}17 & 8 \\ \text { Group: FCM MRD- NGS MRD- } & -20\end{array}$
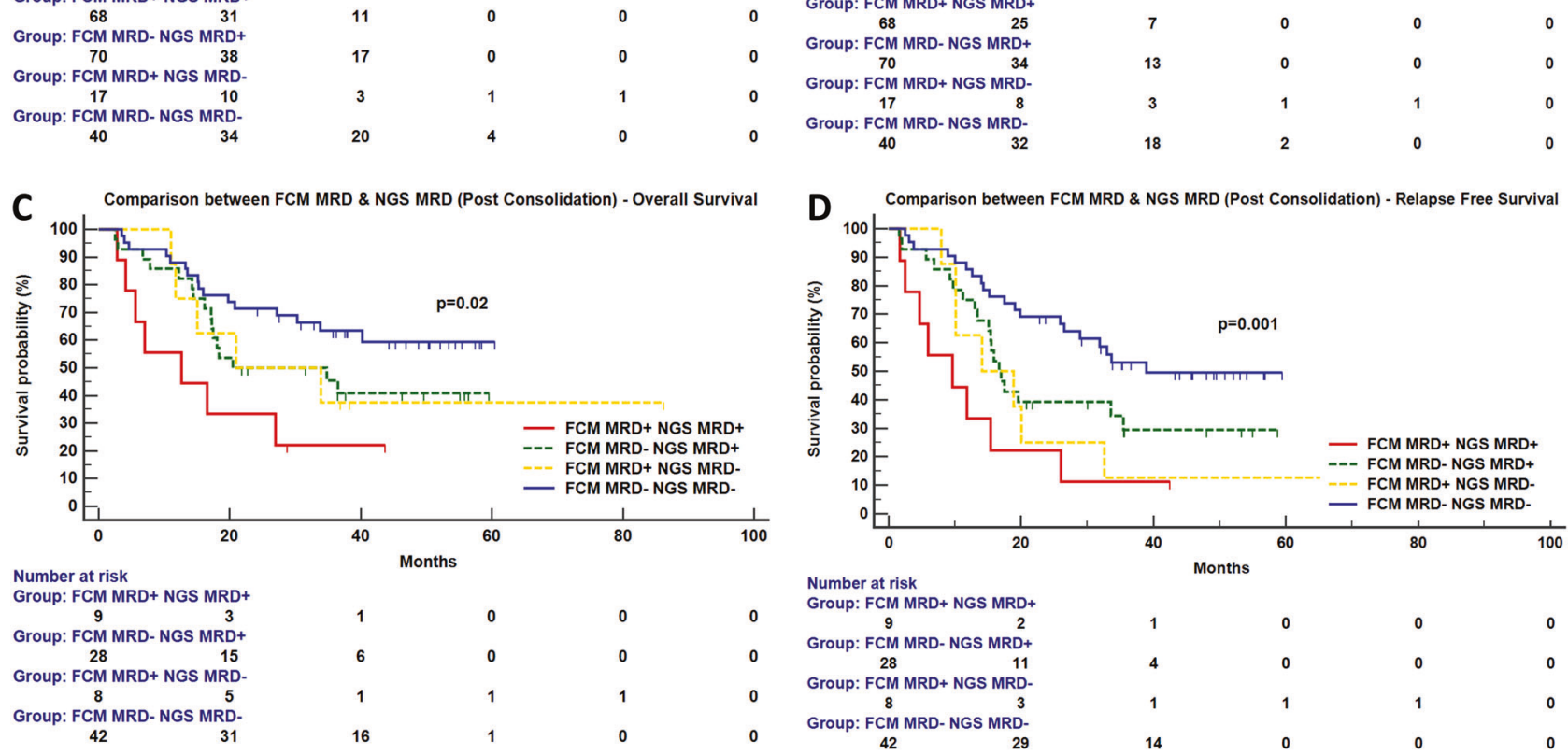

\& NGS MRD (Post Consolidation) - Relapse Free Survival

Fig. 3 Comparison between FCM and NGS-MRD. The clinical relevance detection of MRD during complete remission when measured by FCM or error-corrected NGS at post induction $(\mathbf{A}, \mathbf{B})$ and post consolidation time points $(\mathbf{C}, \mathbf{D})$.

Analysis of relapsed patients $(n=7)$ revealed that three samples had lower coverage (mean consensus coverage$8363 x$ ). In these cases, MRD detection could have been inaccurate due to suboptimal sensitivity. Two out of the remaining three discrepant cases were NGS-MRD ${ }^{-}$at the end of consolidation but were NGS-MRD ${ }^{+}$at the end of induction. A discrepant NGS-MRD result is unlikely to have therapeutic implications in these two patients.
We demonstrate that patients who are NGS-MRD ${ }^{-}$at the end of induction are likely to have a superior outcome as compared to patients who subsequently become NGS$\mathrm{MRD}^{-}$(Supplementary Fig. 12). We have previously demonstrated that NPM1 NGS-MRD values are comparable when simultaneously measured from the blood and bone marrow [21]. We observed a good correlation with a minimal loss of sensitivity when MRD measurements were 
Table 4 Multivariate cox analysis for the presence of FLT3-ITD, RUNX1 mutation, FCM-MRD and NGS-MRD at post induction and post consolidation time points for OS, RFS, and CIR.

\begin{tabular}{|c|c|c|c|c|}
\hline \multicolumn{5}{|l|}{ Multivariate cox analysis } \\
\hline & \multicolumn{2}{|c|}{ Overall survival (OS) } & \multicolumn{2}{|c|}{ Relapse free survival (RFS) } \\
\hline & HR $(95 \% \mathrm{CI})$ & $P$ & HR $(95 \% \mathrm{CI})$ & $P$ \\
\hline FLT3-ITD mutation & $2.41(1.43-4.09)$ & 0.001 & $2.30(1.42-3.71)$ & 0.0006 \\
\hline RUNX1 mutation & $2.76(1.25-6.06)$ & 0.012 & $2.14(0.99-4.61)$ & 0.05 \\
\hline Poor cytogenetic risk & $1.46(0.73-2.90)$ & 0.276 & $1.42(0.74-2.70)$ & 0.284 \\
\hline PI FCM-MRD positive & $1.88(1.22-2.91)$ & 0.004 & $1.70(1.15-2.49)$ & 0.007 \\
\hline \multirow[t]{3}{*}{ PI NGS-MRD positive } & $1.99(1.16-3.40)$ & 0.012 & $2.01(1.26-3.18)$ & 0.003 \\
\hline & \multicolumn{4}{|c|}{ Cumulative incidence of relapse (CIR) } \\
\hline & \multicolumn{3}{|l|}{ HR $(95 \% \mathrm{CI})$} & $P$ \\
\hline FLT3-ITD mutation & \multicolumn{3}{|l|}{$1.64(0.84-3.20)$} & 0.14 \\
\hline RUNX1 mutation & \multicolumn{3}{|l|}{$3.63(1.31-10.0)$} & 0.013 \\
\hline Poor cytogenetic risk & \multicolumn{3}{|l|}{$0.80(0.34-1.86)$} & 0.6 \\
\hline PI FCM-MRD positive & \multicolumn{3}{|l|}{$1.89(1.17-3.03)$} & 0.009 \\
\hline \multirow[t]{3}{*}{ PI NGS-MRD positive } & \multicolumn{3}{|l|}{$2.32(1.28-4.20)$} & 0.006 \\
\hline & \multicolumn{2}{|c|}{ Overall survival (OS) } & \multicolumn{2}{|c|}{ Relapse free survival (RFS) } \\
\hline & HR $(95 \% \mathrm{CI})$ & $P$ & HR $(95 \% \mathrm{CI})$ & $P$ \\
\hline FLT3-ITD mutation & $3.36(1.56-7.23)$ & 0.002 & $3.60(1.75-7.37)$ & 0.0005 \\
\hline RUNX1 mutation & $2.28(0.62-8.42)$ & 0.215 & $1.40(0.39-4.94)$ & 0.599 \\
\hline Poor cytogenetic risk & $0.65(0.23-1.81)$ & 0.416 & $0.86(0.35-2.10)$ & 0.745 \\
\hline PC FCM-MRD positive & $1.92(0.95-3.87)$ & 0.07 & $2.63(1.41-4.90)$ & 0.002 \\
\hline \multirow[t]{3}{*}{ PC NGS-MRD positive } & $1.61(0.86-3.03)$ & 0.136 & $1.65(0.94-2.89)$ & 0.08 \\
\hline & \multicolumn{4}{|c|}{ Cumulative incidence of relapse (CIR) } \\
\hline & \multicolumn{3}{|l|}{ HR $(95 \% \mathrm{CI})$} & $P$ \\
\hline FLT3-ITD mutation & \multicolumn{3}{|l|}{$2.85(1.07-7.61)$} & 0.04 \\
\hline RUNX1 mutation & \multicolumn{3}{|l|}{$5.09(0.94-27.5)$} & 0.06 \\
\hline Poor cytogenetic risk & \multicolumn{3}{|l|}{$1.24(0.40-3.83)$} & 0.7 \\
\hline PC FCM-MRD positive & \multicolumn{3}{|l|}{$3.90(1.90-8.03)$} & $<0.001$ \\
\hline PC NGS-MRD positive & \multicolumn{3}{|l|}{$1.71(0.86-3.38)$} & 0.12 \\
\hline
\end{tabular}

$O S$ overall survival, $R F S$ relapse free survival, $C I R$ cumulative incidence of relapse, $P I$ post induction, $P C$ post induction. made in the blood (median of $0.4 \log$ difference of VAF levels when compared to BM) [21]. In this manuscript, we demonstrate that a blood sample may be acceptable for NGS-MRD when BM sampling is unfeasible.

Previously Jongen-Lavrencic and colleagues have demonstrated clinical utility of NGS to detect MRD in AML by using computational error correction to mitigate sequencing errors [27]. Such an approach, although easy to implement, discounts for batch effects and variability that occurs because of library clustering and batch-dependent PCR artefacts [32]. In that context, to the best of our knowledge, this is the first study to determine the clinical importance of (error-corrected, panel-based) NGS-MRD in AML treated with conventional therapies. Although our NGS-MRD strategy works in a majority of AML, we were curious about the genetic basis of cases ( $n=65$ out of 393) in which this strategy did not work. The cytogenetic and mutational landscape can be seen in Supplementary Fig. 18 and their outcome is detailed in supplementary methods. Many of these patients demonstrated favorable cytogenetic risk (49.2\%) and nearly half did not show presence of any mutation at diagnosis $(n=29 ; 44.6 \%)$. Insight into rest of the cases revealed $A S X L 2$ as a recurrently mutated gene $(n=8,12.3 \%)$. Incorporation of ASXL2 in future iterations of our panel will help in increasing the breadth of our approach. Alternative MRD monitoring approaches such as qPCR or UMI-based RNA sequencing [24, 25] to monitor chimeric gene fusions should be considered in favorable risk AML. This would be expected to increase the applicability of MRD detection to $>90 \%$ of all AML patients in our cohort.

Consistent with previous reports, we find that in some patients, mutations in DTA genes are present at high VAF at MRD time points (Fig. 1) indicating an origin from an ancestral clone possibly originating from clonal haematopoesis [27, 34, 42]. In our series, a total of 33 patients had 
DTA mutations which were trackable by MRD panel (including three with a sole DTA mutation). Of these, 21 patients were NGS-MRD ${ }^{+}$with persistent DTA mutations. The decision to label a patient as NGS-MRD ${ }^{+}$in 19 patients was not based on the presence of DTA mutation but was made on other persistent non-DTA mutations. In two patients as no other mutation was trackable, the decision to label the patient as NGS-MRD ${ }^{+}$was made based on a persistent TET2 clone. The clinical relevance of NGS-MRD after exclusion of DTA mutations can be seen in Supplementary Fig. 19. No patient was considered as NGS-MRD ${ }^{+}$ based on persistence of DTA mutation at the end of consolidation.

Unlike amplicon-based [28, 34] approaches, the advantage of a smMIPS-based capture includes a stable panel which can be used across a spectrum of cases and no susceptibility to allelic skew or PCR-induced errors prior to incorporation of the UMI barcode. Disadvantages of smMIPS include poor performance for GC rich genes such as CEBPA gene and inability to capture low yield or poor quality of DNA (a problem not infrequently seen with MRD samples). The library preparation process is relatively low cost in nature and the overall process has a realistic turnaround time of 5-7 days. Our observation is that sensitivity in the clinic for most mutations is close to $0.1 \% \mathrm{VAF}$. A higher sensitivity can be obtained for complex indels such as NPM1 and FLT3-ITD. This level of sensitivity $(0.03 \%$ VAF) for NPM1 mutated AML is at least one log lower than what is possible by RNA-based qPCR assays [17]. However, advantages over qPCR include the ability to monitor any NPM1 mutation subtype in a single assay with uniform assay performance characteristics across all NPM1 subtypes [44].

Improvements with sensitivity may be possible through duplex-sequencing based methods albeit at a much higher cost of sequencing [30]. Based on this data we find that mutations in NPM1, FLT3, NRAS, KIT, IDH1, IDH2, WT1, RUNX1, GATA2, U2AF1, and PHF6 were most helpful in considering a patient as NGS-MRD ${ }^{+}$(Supplementary Fig. 20). In our study, a vast majority (75.7\%) of mutations monitored in patients with favorable cytogenetic risk include signaling pathway mutations (in FLT3, KIT, NRAS and KRAS genes) which could be susceptible to clonal evolution with targeted therapy. A limitation of our study is lack of orthogonal comparison with standard qPCR MRD tests for detection of fusion transcripts such as RUNX1RUNX1T1 or CBFB-MYH11.

To conclude, we demonstrate that panel-based errorcorrected NGS-MRD is clinically relevant and synergistic in application to FCM-based AML MRD monitoring.

Acknowledgements We are grateful for the training imparted to $D r$. Nikhil Patkar in the field genome sequencing technologies by Dr. David
Wu, Dr. Stephen Salipante and Dr. Brent Wood in the Department of Laboratory Medicine, University of Washington, USA \& Salipante Lab, University of Washington. We are greatful to Dr. Jay Shendure (Department of Genome Sciences, University of Washington) for agreeing to be an the external sponsor for Wellcome Trust/DBT India Alliance Fellowship. We are gratetful to Dr. Christopher Hourigan for critically reviewing this manuscript and providing helpful comments and suggestions.

Funding This work was supported by the Wellcome Trust/DBT India Alliance Fellowship [grant number IA/CPHI/14/1/501485] awarded to Dr. Nikhil Patkar.

Author contributions NP designed the study, conducted research, analyzed, interpreted the data, and wrote the manuscript. AFS, CK, RS, PB, SR, SJ, SC, RK conducted research and analyzed data. DS, SG, GC, ND, PT, PGS, SG conducted research and analyzed data. BB, $\mathrm{HM}, \mathrm{NK}, \mathrm{MS}$ recruited patients and analyzed data.

\section{Compliance with ethical standards}

Conflict of interest The authors declare that they have no conflict of interest.

Publisher's note Springer Nature remains neutral with regard to jurisdictional claims in published maps and institutional affiliations.

Open Access This article is licensed under a Creative Commons Attribution 4.0 International License, which permits use, sharing, adaptation, distribution and reproduction in any medium or format, as long as you give appropriate credit to the original author(s) and the source, provide a link to the Creative Commons license, and indicate if changes were made. The images or other third party material in this article are included in the article's Creative Commons license, unless indicated otherwise in a credit line to the material. If material is not included in the article's Creative Commons license and your intended use is not permitted by statutory regulation or exceeds the permitted use, you will need to obtain permission directly from the copyright holder. To view a copy of this license, visit http://creativecommons. org/licenses/by/4.0/.

\section{References}

1. Estey E, Dohner H. Acute myeloid leukaemia. Lancet. 2006;368:1894-907.

2. Papaemmanuil E, Gerstung M, Bullinger L, Gaidzik VI, Paschka $\mathrm{P}$, Roberts ND, et al. Genomic classification and prognosis in acute myeloid leukemia. N Engl J Med. 2016;374:2209-21.

3. Stone RM, Mandrekar SJ, Sanford BL, Laumann K, Geyer S, Bloomfield CD, et al. Midostaurin plus chemotherapy for acute myeloid leukemia with a FLT3 mutation. $\mathrm{N}$ Engl J Med. 2017;377:454-64.

4. Stein EM, DiNardo CD, Pollyea DA, Fathi AT, Roboz GJ, Altman JK, et al. Enasidenib in mutant IDH2 relapsed or refractory acute myeloid leukemia. Blood. 2017;130:722-31.

5. Dohner H, Estey E, Grimwade D, Amadori S, Appelbaum FR, Buchner T, et al. Diagnosis and management of AML in adults: 2017 ELN recommendations from an international expert panel. Blood. 2017;129:424-47.

6. Kern W, Bacher U, Haferlach C, Schnittger S, Haferlach T. The role of multiparameter flow cytometry for disease monitoring in AML. Best Pract Res Clin Haematol. 2010;23:379-90. 
7. Walter RB, Gooley TA, Wood BL, Milano F, Fang M, Sorror ML, et al. Impact of pretransplantation minimal residual disease, as detected by multiparametric flow cytometry, on outcome of myeloablative hematopoietic cell transplantation for acute myeloid leukemia. J Clin Oncol. 2011;29:1190-7.

8. Loken MR, Alonzo TA, Pardo L, Gerbing RB, Raimondi SC, Hirsch BA, et al. Residual disease detected by multidimensional flow cytometry signifies high relapse risk in patients with de novo acute myeloid leukemia: a report from Children's Oncology Group. Blood. 2012;120:1581-8.

9. Patkar N, Kakirde C, Bhanshe P, Joshi S, Chaudhary S, Badrinath $\mathrm{Y}$, et al. Utility of immunophenotypic measurable residual disease in adult acute myeloid leukemia-real-world context. Front Oncol. 2019;9:450.

10. San Miguel JF, Vidriales MB, Lopez-Berges C, Diaz-Mediavilla J, Gutierrez N, Canizo C, et al. Early immunophenotypical evaluation of minimal residual disease in acute myeloid leukemia identifies different patient risk groups and may contribute to postinduction treatment stratification. Blood. 2001;98:1746-51.

11. Freeman SD, Virgo P, Couzens S, Grimwade D, Russell N, Hills RK. Prognostic relevance of treatment response measured by flow cytometric residual disease detection in older patients with acute myeloid leukemia. J Clin Oncol. 2013;2013:31.

12. Buccisano F, Maurillo L, Spagnoli A, Del Principe MI, Fraboni D, Panetta $\mathrm{P}$, et al. Cytogenetic and molecular diagnostic characterization combined to postconsolidation minimal residual disease assessment by flow cytometry improves risk stratification in adult acute myeloid leukemia. Blood. 2010;116:2295-303.

13. Venditti A, Buccisano F, Del Poeta G, Maurillo L, Tamburini A, Cox $\mathrm{C}$, et al. Level of minimal residual disease after consolidation therapy predicts outcome in acute myeloid leukemia. Blood. 2000;96:3948-52.

14. Terwijn M, Putten WLJ, Kelder A, Velden VHJ, Brooimans RA, Pabst T. High prognostic impact of flow cytometric minimal residual disease detection in acute myeloid leukemia: data from the HOVON/SAKK AML 42A study. J Clin Oncol. 2013;2013:31.

15. Gabert J, Beillard E, van der Velden VH, Bi W, Grimwade D, Pallisgaard N, et al. Standardization and quality control studies of 'real-time' quantitative reverse transcriptase polymerase chain reaction of fusion gene transcripts for residual disease detection in leukemia - a Europe Against Cancer program. Leukemia. 2003; 17:2318-57.

16. Mencia-Trinchant N, Hu Y, Alas MA, Ali F, Wouters BJ, Lee S, et al. Minimal residual disease monitoring of acute myeloid leukemia by massively multiplex digital PCR in patients with NPM1 mutations. J Mol Diagn. 2017;19:537-48.

17. Ivey A, Hills RK, Simpson MA, Jovanovic JV, Gilkes A, Grech A, et al. Assessment of minimal residual disease in standard-risk AML. N Engl J Med. 2016;374:422-33.

18. Dillon R, Hills R, Freeman S, Potter N, Jovanovic J, Ivey A, et al. Molecular MRD status and outcome after transplantation in NPM1-mutated AML. Blood. 2020;135:680-8.

19. Rucker FG, Agrawal M, Corbacioglu A, Weber D, KappSchwoerer S, Gaidzik VI, et al. Measurable residual disease monitoring in acute myeloid leukemia with $\mathrm{t}(8 ; 21)(\mathrm{q} 22 ; \mathrm{q} 22.1)$ : results from the AML Study Group. Blood. 2019;134:1608-18.

20. Salipante SJ, Fromm JR, Shendure J, Wood BL, Wu D. Detection of minimal residual disease in NPM1-mutated acute myeloid leukemia by next-generation sequencing. Mod Pathol. 2014;27:1438-46.

21. Patkar N, Kodgule R, Kakirde C, Raval G, Bhanshe P, Joshi S, et al. Clinical impact of measurable residual disease monitoring by ultradeep next generation sequencing in NPM1 mutated acute myeloid leukemia. Oncotarget. 2018;9:36613-24.
22. Kohlmann A, Nadarajah N, Alpermann T, Grossmann V, Schindela S, Dicker F, et al. Monitoring of residual disease by nextgeneration deep-sequencing of RUNX1 mutations can identify acute myeloid leukemia patients with resistant disease. Leukemia. 2014;28:129-37.

23. Levis MJ, Perl AE, Altman JK, Gocke CD, Bahceci E, Hill J, et al. A next-generation sequencing-based assay for minimal residual disease assessment in AML patients with FLT3-ITD mutations. Blood Adv. 2018;2:825-31.

24. Dillon LW, Hayati S, Roloff GW, Tunc I, Pirooznia M, Mitrofanova A, et al. Targeted RNA-sequencing for the quantification of measurable residual disease in acute myeloid leukemia. Haematologica. 2019;104:297-304.

25. Patkar N, Bhanshe P, Rajpal S, Joshi S, Chaudhary S, Chatterjee G. et al.NARASIMHA: novel assay based on targeted rna sequencing to identify chimeric gene fusions in hematological malignancies.Blood Cancer J. 2020;10:50

26. Kim T, Moon JH, Ahn JS, Kim YK, Lee SS, Ahn SY, et al. Nextgeneration sequencing-based posttransplant monitoring of acute myeloid leukemia identifies patients at high risk of relapse. Blood. 2018;132:1604-13.

27. Jongen-Lavrencic M, Grob T, Hanekamp D, Kavelaars FG, Al Hinai A, Zeilemaker A, et al. Molecular minimal residual disease in acute myeloid leukemia. N. Engl J Med. 2018;378:1189-99.

28. Press RD, Eickelberg G, Froman A, Yang F, Stentz A, Flatley EM, et al. Next-generation sequencing-defined minimal residual disease before stem cell transplantation predicts acute myeloid leukemia relapse. Am J Hematol. 2019;94:902-12.

29. Klco JM, Miller CA, Griffith M, Petti A, Spencer DH, KetkarKulkarni S, et al. Association between mutation clearance after induction therapy and outcomes in acute myeloid leukemia. JAMA. 2015;314:811-22.

30. Schmitt MW, Kennedy SR, Salk JJ, Fox EJ, Hiatt JB, Loeb LA. Detection of ultra-rare mutations by next-generation sequencing. Proc Natl Acad Sci USA. 2012;109:14508-13.

31. Hiatt JB, Pritchard CC, Salipante SJ, O'Roak BJ, Shendure J. Single molecule molecular inversion probes for targeted, highaccuracy detection of low-frequency variation. Genome Res. 2013;23:843-54.

32. Yoest JM, Shirai CL, Duncavage EJ. Sequencing-based measurable residual disease testing in acute myeloid leukemia. Front Cell Dev Biol. 2020;8:249.

33. Duncavage EJ, Jacoby MA, Chang GS, Miller CA, Edwin N, Shao J, et al. Mutation clearance after transplantation for myelodysplastic syndrome. N Engl J Med. 2018;379:1028-41.

34. Thol F, Gabdoulline R, Liebich A, Klement P, Schiller J, Kandziora $\mathrm{C}$, et al. Measurable residual disease monitoring by NGS before allogeneic hematopoietic cell transplantation in AML. Blood. 2018;132:1703-13.

35. Waalkes A, Penewit K, Wood BL, Wu D, Salipante SJ. Ultrasensitive detection of acute myeloid leukemia minimal residual disease using single molecule molecular inversion probes. Haematologica. 2017;102:1549-57.

36. Patkar N, Shaikh AF, Kakirde C, Nathany S, Ramesh H, Bhanshe $\mathrm{P}$, et al. A novel machine-learning-derived genetic score correlates with measurable residual disease and is highly predictive of outcome in acute myeloid leukemia with mutated NPM1. Blood cancer J. 2019;9:79.

37. Blatte TJ, Schmalbrock LK, Skambraks S, Lux S, Cocciardi S, Dolnik A, et al. getITD for FLT3-ITD-based MRD monitoring in AML. Leukemia. 2019;33:2535-9.

38. Shaikh AF, Kakirde C, Dhamne C, Bhanshe P, Joshi S, Chaudhary S. et al. Machine learning derived genomics driven prognostication for acute myeloid leukemia with RUNX1RUNX1T1. Leuk lymphoma. 2020;61(13):3154-60. 
39. Scrucca L, Santucci A, Aversa F. Competing risk analysis using $\mathrm{R}$ : an easy guide for clinicians. Bone marrow Transplant. 2007;40:381-7.

40. Dignam JJ, Zhang Q, Kocherginsky M. The use and interpretation of competing risks regression models. Clin Cancer Res. 2012;18:2301-8.

41. MedCalc. Diagnostic test evaluation calculator. 2020.

42. Hourigan CS, Dillon LW, Gui G, Logan BR, Fei M, Ghannam J, et al. Impact of conditioning intensity of allogeneic transplantation for acute myeloid leukemia with genomic evidence of residual disease. J Clin Oncol. 2020;38:1273-83.

43. Schuurhuis GJ, Heuser M, Freeman S, Béné MC, Buccisano F, Cloos $\mathrm{J}$, et al. Minimal/measurable residual disease in AML: a consensus document from the European Leukemia Net MRD Working Party. Blood. $2018 ; 131: 1275-91$.

44. Schnittger S, Kern W, Tschulik C. Minimal residual disease levels assessed by NPM1 mutation-specific RQ-PCR provide important prognostic information in AML. Blood. 2009;114:2220-31. 